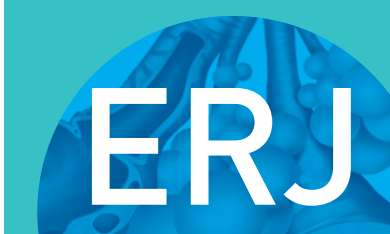

open research

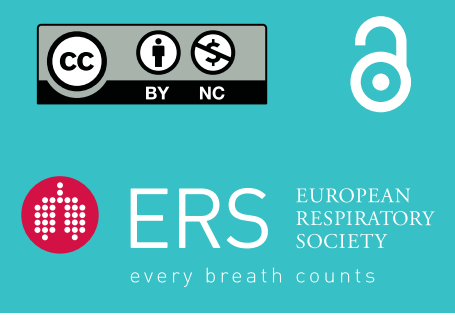

\section{Trends of multidrug-resistant tuberculosis clustering in Portugal}

\author{
To the Editor:
}

Multidrug-resistant (MDR) tuberculosis (TB) represent a major threat for global TB control. In 2017, the World Health Organization estimated 460000 cases of MDR-TB, of which $8.5 \%$ were also extensively drug-resistant (XDR) cases [1]. In Portugal, over the last decade, the decreasing tendency of TB cases is about $7 \%$ per year, and the proportion of MDR-TB cases remains steadily around $1 \%$ of the total TB cases. In 2017, the preliminary report of the Portuguese national TB programme reported 1607 new cases of pulmonary TB, with 12 MDR-TB cases [2].

Since 2014, there have been specific centres for the diagnosis, consultancy, monitoring and treatment of the MDR/XDR-TB cases. Besides providing a clinical approach, these centres also aim to monitor these resistant cases, linking the epidemiological survey performed within the community by public health authorities [3] and systematic molecular genotyping performed by the National Reference Laboratory (NRL). Since the Portuguese NRL receives all the strains isolated from all the MDR-TB patients from Portugal (mandatory since 2007) [4], this approach could allow a very good correlation between the genetic and epidemiological information in order to detect both the resistance profiles, as there are possible relationships between strains due to the occurrence of ongoing transmission $[5,6]$.

In this study, we intended to analyse the MDR-TB clustering rate in Portugal.

From a total of $78 \mathrm{MDR} / \mathrm{XDR}-\mathrm{TB}$ strains identified and notified in the country during 2014-2017, 71 (91.0\%) were available for molecular analysis. From these 78 strains, seven were not available for further analysis due to contamination of the culture or MDR diagnosis based only on molecular biology methodologies (GeneXpert or other line-probe assays). The drug susceptibility profiles are described in table 1.

For each strain, 24-loci MIRU-VNTR (mycobacterial interspersed repetitive units - variable number of tandem repeats) genotyping was performed by standardised protocols using a MIRU-VNTR typing kit, according to the manufacturer's instructions (GenoScreen, Lille, France). Dendrograms were constructed using the online free software MIRU-VNTRplus (https://miru-vntrplus.org/MIRU/miruinfo.faces). A molecular cluster was defined whenever different strains shared the exact MIRU-VNTR profile. All clusters identified were further analysed with the available epidemiological data.

The majority of the MDR-TB cases were male (75.6\%) with a median age of 44.3 years (minimum 15 and maximum 75 years). Most of these cases were notified in the Lisbon and Tagus Valley (LTV) region $(64.0 \%)$ and the North region (23.1\%). XDR-TB cases were identified in 15 cases (19.2\%), of which 86.7\% were from the LTV region (table 1).

Using MIRU-VNTR, seven different clusters were identified (table 1), ranging from two to 14 strains. Overall, the proportion of MDR-TB cases attributable to recent transmission in the study period (2014-2017), on the basis of genetic data, was 63.4\% (45 out of 71).

From the analysis of the molecular data, we observed a decreasing tendency of the cases that can be potentially related to recent transmission. In fact, in 2014, we found six clusters ranging from two to four strains, corresponding to a clustering rate of $72.7 \%$. The major cluster was from strains isolated in the

@ERSpublications

Analysis of MDR-TB rates showed consistent decreases in cases and clustering rates but did not establish valid relationships with the epidemiological information collected by the public health authorities http://ow.ly/3BnN30nxqW3

Cite this article as: Macedo R, Duarte R. Trends of multidrug-resistant tuberculosis clustering in Portugal. ERJ Open Res 2019; 5: 00151-2018 [https://doi.org/10.1183/23120541.00151-2018].

Copyright $\odot$ ERS 2019. This article is open access and distributed under the terms of the Creative Commons Attribution Non-Commercial Licence 4.0. 
TABLE 1 Microbiological and demographic characteristics of the patients enrolled in the study

\begin{tabular}{|c|c|c|c|c|c|c|c|c|c|c|c|c|c|c|c|c|c|c|c|c|c|}
\hline Lab no. & $\begin{array}{c}\text { Diagnosis } \\
\text { year }\end{array}$ & $\begin{array}{c}\text { Exclusion } \\
\text { reason }\end{array}$ & Sex & $\begin{array}{l}\text { Age } \\
\text { years }\end{array}$ & $\begin{array}{l}\text { Region of } \\
\text { isolation }\end{array}$ & $\begin{array}{c}\text { MDR/ } \\
\text { XDR-TB }\end{array}$ & STR & INH & RMP & EMB & PZA & AMI & CAP & ETI & MOX & OFL & LIN & KAN & CIC & PAS & $\begin{array}{c}\text { Cluster } \\
\text { no. }\end{array}$ \\
\hline \multirow[t]{3}{*}{ S199228 } & 2014 & & $M$ & 42 & LTV & XDR & $\mathrm{R}$ & $\mathrm{R}$ & $\mathrm{R}$ & $\mathrm{R}$ & $\mathrm{R}$ & $\mathrm{R}$ & $\mathrm{R}$ & $\mathrm{R}$ & $\mathrm{R}$ & $\mathrm{R}$ & S & S & S & S & \\
\hline & 2014 & $\begin{array}{l}\text { No culture } \\
\text { isolation }\end{array}$ & $M$ & 43 & LTV & XDR & $\mathrm{R}$ & $\mathrm{R}$ & $\mathrm{R}$ & $\mathrm{R}$ & $\mathrm{R}$ & $\mathrm{R}$ & $\mathrm{R}$ & $\mathrm{R}$ & $\mathrm{R}$ & $\mathrm{R}$ & S & S & S & S & \\
\hline & 2014 & Only LPA & M & 47 & LTV & MDR & NA & $\mathrm{R}$ & $\mathrm{R}$ & NA & NA & NA & NA & NA & NA & NA & NA & NA & NA & NA & \\
\hline TB25429 & 2014 & & $M$ & 41 & LTV & MDR & $\mathrm{R}$ & $\mathrm{R}$ & $\mathrm{R}$ & $\mathrm{R}$ & $\mathrm{R}$ & $\mathrm{S}$ & $\mathrm{S}$ & $\mathrm{R}$ & $\mathrm{S}$ & $\mathrm{S}$ & $\mathrm{S}$ & $\mathrm{S}$ & $\mathrm{S}$ & $\mathrm{S}$ & 6 \\
\hline P1595 & 2014 & & $M$ & 37 & LTV & MDR & $\mathrm{R}$ & $\mathrm{R}$ & $\mathrm{R}$ & S & $\mathrm{R}$ & S & S & $\mathrm{R}$ & $\mathrm{S}$ & S & S & S & S & S & 1 \\
\hline P1279 & 2014 & & $\mathrm{~F}$ & 31 & LTV & MDR & $\mathrm{R}$ & $\mathrm{R}$ & $\mathrm{R}$ & $\mathrm{R}$ & $\mathrm{R}$ & S & S & $\mathrm{R}$ & $\mathrm{S}$ & S & S & $\mathrm{R}$ & S & S & \\
\hline S207797 & 2014 & & M & 53 & LTV & MDR & $\mathrm{R}$ & $\mathrm{R}$ & $\mathrm{R}$ & S & $\mathrm{R}$ & S & S & $\mathrm{R}$ & $\mathrm{S}$ & S & S & S & S & S & 2 \\
\hline P1428 & 2014 & & M & 48 & LTV & MDR & $\mathrm{R}$ & $\mathrm{R}$ & $\mathrm{R}$ & S & $\mathrm{R}$ & S & S & $\mathrm{R}$ & $\mathrm{S}$ & S & S & S & S & S & 1 \\
\hline P1378 & 2014 & & M & 50 & LTV & XDR & $\mathrm{R}$ & $\mathrm{R}$ & $\mathrm{R}$ & $\mathrm{R}$ & $\mathrm{R}$ & S & S & $\mathrm{R}$ & $\mathrm{R}$ & $\mathrm{R}$ & S & $\mathrm{R}$ & S & S & 3 \\
\hline P163 & 2014 & & $M$ & 53 & LTV & XDR & $\mathrm{R}$ & $\mathrm{R}$ & $\mathrm{R}$ & $\mathrm{R}$ & $\mathrm{R}$ & $\mathrm{R}$ & S & $\mathrm{R}$ & $\mathrm{R}$ & $\mathrm{R}$ & S & $\mathrm{R}$ & S & S & 3 \\
\hline TB24818 & 2014 & & $\mathrm{~F}$ & 32 & LTV & MDR & $\mathrm{R}$ & $\mathrm{R}$ & $\mathrm{R}$ & $\mathrm{R}$ & $\mathrm{S}$ & S & S & S & $\mathrm{S}$ & S & S & S & S & S & 7 \\
\hline P291 & 2014 & & M & 28 & LTV & MDR & $\mathrm{R}$ & $\mathrm{R}$ & $\mathrm{R}$ & $\mathrm{R}$ & $\mathrm{S}$ & S & S & S & $\mathrm{S}$ & S & S & S & S & S & \\
\hline TB25274 & 2014 & & $M$ & 37 & LTV & XDR & $\mathrm{R}$ & $\mathrm{R}$ & $\mathrm{R}$ & $\mathrm{R}$ & $\mathrm{R}$ & S & S & $\mathrm{R}$ & $\mathrm{S}$ & $\mathrm{R}$ & S & $\mathrm{R}$ & $\mathrm{R}$ & S & \\
\hline S211891 & 2014 & & M & 36 & LTV & MDR & $\mathrm{R}$ & $\mathrm{R}$ & $\mathrm{R}$ & $\mathrm{R}$ & $\mathrm{S}$ & S & S & $\mathrm{R}$ & $\mathrm{S}$ & S & S & S & S & S & 7 \\
\hline P1599 & 2014 & & $M$ & 43 & LTV & MDR & $\mathrm{R}$ & $\mathrm{R}$ & $\mathrm{R}$ & $\mathrm{R}$ & $\mathrm{S}$ & S & S & S & $\mathrm{S}$ & S & S & S & S & S & 6 \\
\hline P187 & 2014 & & $M$ & 58 & LTV & MDR & $\mathrm{R}$ & $\mathrm{R}$ & $\mathrm{R}$ & $\mathrm{R}$ & $\mathrm{R}$ & S & S & $\mathrm{R}$ & $\mathrm{S}$ & S & S & S & S & S & 2 \\
\hline P88 & 2014 & & $M$ & 63 & North & MDR & $\mathrm{R}$ & $\mathrm{R}$ & $\mathrm{R}$ & $\mathrm{S}$ & $\mathrm{S}$ & S & S & S & S & S & S & S & S & S & 4 \\
\hline P423 & 2014 & & $\mathrm{~F}$ & 75 & North & MDR & $\mathrm{R}$ & $\mathrm{R}$ & $\mathrm{R}$ & S & $\mathrm{S}$ & S & S & S & S & S & S & S & S & S & 4 \\
\hline P292 & 2014 & & $\mathrm{~F}$ & 41 & North & MDR & $\mathrm{R}$ & $\mathrm{R}$ & $\mathrm{R}$ & S & S & S & S & S & $\mathrm{S}$ & S & S & S & S & $\mathrm{S}$ & 4 \\
\hline P1536 & 2014 & & $M$ & 23 & North & MDR & $\mathrm{R}$ & $\mathrm{R}$ & $\mathrm{R}$ & S & S & S & S & S & $\mathrm{S}$ & S & S & S & S & S & 4 \\
\hline P729 & 2014 & & $M$ & 47 & North & MDR & $\mathrm{R}$ & $\mathrm{R}$ & $\mathrm{R}$ & S & S & S & S & S & $\mathrm{S}$ & $S$ & $S$ & $S$ & S & S & \\
\hline P356 & 2014 & & $M$ & 42 & North & MDR & S & $\mathrm{R}$ & $\mathrm{R}$ & S & S & S & S & S & $\mathrm{S}$ & S & $S$ & $\mathrm{~S}$ & $S$ & $\mathrm{~S}$ & \\
\hline P92 & 2014 & & $M$ & 59 & Centre & MDR & $\mathrm{R}$ & $\mathrm{R}$ & $\mathrm{R}$ & S & S & $S$ & $S$ & $\mathrm{R}$ & $\mathrm{S}$ & S & S & $\mathrm{S}$ & S & S & 2 \\
\hline \multirow[t]{2}{*}{ P340 } & 2014 & & $M$ & 48 & Centre & XDR & $\mathrm{R}$ & $\mathrm{R}$ & $\mathrm{R}$ & $\mathrm{R}$ & $\mathrm{S}$ & $\mathrm{R}$ & S & $\mathrm{R}$ & S & $\mathrm{R}$ & S & $\mathrm{R}$ & S & S & 3 \\
\hline & 2015 & Only LPA & $M$ & 31 & LTV & MDR & NA & $\mathrm{R}$ & $\mathrm{R}$ & NA & NA & NA & NA & NA & NA & NA & NA & NA & NA & NA & \\
\hline P2624 & 2015 & & $M$ & 28 & LTV & MDR & $\mathrm{R}$ & $\mathrm{R}$ & $\mathrm{R}$ & $\mathrm{R}$ & $\mathrm{S}$ & $\mathrm{S}$ & $\mathrm{S}$ & $\mathrm{R}$ & $\mathrm{S}$ & $\mathrm{S}$ & $\mathrm{S}$ & $\mathrm{S}$ & $\mathrm{S}$ & $\mathrm{S}$ & 7 \\
\hline P1928 & 2015 & & $M$ & 61 & LTV & MDR & $\mathrm{R}$ & $\mathrm{R}$ & $\mathrm{R}$ & $\mathrm{R}$ & $\mathrm{R}$ & S & S & $\mathrm{R}$ & $\mathrm{S}$ & S & S & S & $S$ & S & 1 \\
\hline P1229 & 2015 & & $M$ & 39 & LTV & XDR & $\mathrm{R}$ & $\mathrm{R}$ & $\mathrm{R}$ & $\mathrm{R}$ & $\mathrm{R}$ & $\mathrm{R}$ & $\mathrm{R}$ & $\mathrm{R}$ & $\mathrm{R}$ & $\mathrm{R}$ & S & $\mathrm{R}$ & $S$ & S & 1 \\
\hline P1876 & 2015 & & $M$ & 55 & LTV & XDR & $\mathrm{R}$ & $\mathrm{R}$ & $\mathrm{R}$ & $\mathrm{R}$ & $\mathrm{R}$ & $\mathrm{R}$ & S & $\mathrm{R}$ & $\mathrm{R}$ & $\mathrm{R}$ & S & $\mathrm{R}$ & S & S & 3 \\
\hline P1926 & 2015 & & M & 52 & LTV & XDR & $\mathrm{R}$ & $\mathrm{R}$ & $\mathrm{R}$ & $\mathrm{R}$ & $\mathrm{R}$ & $\mathrm{R}$ & $\mathrm{R}$ & $\mathrm{R}$ & $\mathrm{R}$ & $\mathrm{R}$ & S & $\mathrm{R}$ & S & $\mathrm{S}$ & 1 \\
\hline P2829 & 2015 & & M & 44 & LTV & MDR & S & $\mathrm{R}$ & $\mathrm{R}$ & S & $\mathrm{S}$ & S & S & $\mathrm{R}$ & $\mathrm{S}$ & S & S & S & S & $\mathrm{S}$ & 1 \\
\hline P2184 & 2015 & & M & 75 & LTV & MDR & $\mathrm{R}$ & $\mathrm{R}$ & $\mathrm{R}$ & $\mathrm{R}$ & $\mathrm{R}$ & $\mathrm{R}$ & $\mathrm{R}$ & $\mathrm{R}$ & S & S & S & $\mathrm{R}$ & S & S & 1 \\
\hline P1994 & 2015 & & $M$ & 41 & LTV & MDR & $\mathrm{R}$ & $\mathrm{R}$ & $\mathrm{R}$ & S & $\mathrm{R}$ & S & S & $\mathrm{R}$ & $\mathrm{S}$ & S & S & $\mathrm{S}$ & S & S & 2 \\
\hline P2058 & 2015 & & $M$ & 57 & LTV & MDR & $\mathrm{R}$ & $\mathrm{R}$ & $\mathrm{R}$ & S & $\mathrm{R}$ & S & S & $\mathrm{R}$ & $\mathrm{S}$ & S & S & S & $S$ & S & \\
\hline TB24737 & 2015 & & $M$ & 44 & LTV & XDR & $\mathrm{R}$ & $\mathrm{R}$ & $\mathrm{R}$ & $\mathrm{R}$ & $\mathrm{R}$ & $\mathrm{R}$ & $\mathrm{R}$ & S & $\mathrm{R}$ & $\mathrm{R}$ & S & $\mathrm{R}$ & S & $\mathrm{R}$ & \\
\hline P1585 & 2015 & & $\mathrm{~F}$ & 42 & LTV & MDR & $\mathrm{R}$ & $\mathrm{R}$ & $\mathrm{R}$ & $\mathrm{R}$ & S & S & S & $\mathrm{R}$ & $\mathrm{S}$ & S & S & S & S & S & 3 \\
\hline P2354 & 2015 & & $\mathrm{~F}$ & 27 & LTV & MDR & $\mathrm{R}$ & $\mathrm{R}$ & $\mathrm{R}$ & $\mathrm{R}$ & $\mathrm{R}$ & $\mathrm{R}$ & $\mathrm{R}$ & S & $\mathrm{S}$ & S & S & $\mathrm{R}$ & S & S & \\
\hline \multirow[t]{2}{*}{ P2471 } & 2015 & & M & 34 & LTV & MDR & $\mathrm{R}$ & $\mathrm{R}$ & $\mathrm{R}$ & $\mathrm{R}$ & $\mathrm{S}$ & S & S & $\mathrm{R}$ & $\mathrm{S}$ & S & S & $\mathrm{S}$ & S & $\mathrm{S}$ & 3 \\
\hline & 2015 & Only LPA & $\mathrm{F}$ & 32 & LTV & MDR & NA & $\mathrm{R}$ & $\mathrm{R}$ & NA & NA & NA & NA & NA & NA & NA & NA & NA & NA & NA & \\
\hline P884 & 2015 & & $M$ & 61 & LTV & MDR & $\mathrm{R}$ & $\mathrm{R}$ & $\mathrm{R}$ & $\mathrm{S}$ & $\mathrm{R}$ & $\mathrm{S}$ & $\mathrm{S}$ & $\mathrm{R}$ & $\mathrm{S}$ & $\mathrm{S}$ & $\mathrm{S}$ & $\mathrm{S}$ & $S$ & $\mathrm{~S}$ & 2 \\
\hline P2579 & 2015 & & $M$ & 35 & LTV & MDR & $\mathrm{R}$ & $\mathrm{R}$ & $\mathrm{R}$ & $\mathrm{R}$ & $\mathrm{R}$ & $\mathrm{S}$ & S & $\mathrm{R}$ & $\mathrm{S}$ & S & S & $\mathrm{S}$ & S & S & \\
\hline P2452 & 2015 & & M & 57 & North & MDR & $\mathrm{R}$ & $\mathrm{R}$ & $\mathrm{R}$ & S & S & S & S & $\mathrm{R}$ & $\mathrm{S}$ & S & S & $\mathrm{S}$ & S & $\mathrm{s}$ & 3 \\
\hline P982 & 2015 & & $M$ & 37 & North & MDR & $\mathrm{R}$ & $\mathrm{R}$ & $\mathrm{R}$ & $\mathrm{R}$ & $\mathrm{R}$ & S & S & $\mathrm{S}$ & $\mathrm{S}$ & $\mathrm{S}$ & S & $\mathrm{S}$ & S & S & \\
\hline
\end{tabular}


TABLE 1 Continued

\begin{tabular}{|c|c|c|c|c|c|c|c|c|c|c|c|c|c|c|c|c|c|c|c|c|c|}
\hline Lab no. & $\begin{array}{l}\text { Diagnosis } \\
\text { year }\end{array}$ & $\begin{array}{l}\text { Exclusion } \\
\text { reason }\end{array}$ & Sex & $\begin{array}{l}\text { Age } \\
\text { years }\end{array}$ & $\begin{array}{l}\text { Region of } \\
\text { isolation }\end{array}$ & $\begin{array}{c}\text { MDR/ } \\
\text { XDR-TB }\end{array}$ & STR & INH & RMP & EMB & PZA & AMI & CAP & ETI & MOX & OFL & LIN & KAN & CIC & PAS & $\begin{array}{c}\text { Cluster } \\
\text { no. }\end{array}$ \\
\hline P1880 & 2015 & & $M$ & 58 & North & MDR & $\mathrm{R}$ & $\mathrm{R}$ & $\mathrm{R}$ & S & S & S & S & S & S & S & S & $\mathrm{R}$ & S & S & \\
\hline P2353 & 2015 & & M & 21 & $\begin{array}{l}\text { Island of } \\
\text { Madeira }\end{array}$ & MDR & S & $\mathrm{R}$ & $\mathrm{R}$ & $\mathrm{R}$ & $\mathrm{S}$ & $\mathrm{S}$ & $\mathrm{S}$ & $\mathrm{R}$ & $\mathrm{S}$ & $\mathrm{S}$ & S & S & $\mathrm{S}$ & $\mathrm{S}$ & 5 \\
\hline \multirow[t]{2}{*}{ P27 } & 2015 & & M & 44 & Algarve & MDR & S & $\mathrm{R}$ & $\mathrm{R}$ & S & $\mathrm{S}$ & S & S & $\mathrm{R}$ & S & S & S & S & S & S & \\
\hline & 2015 & Only LPA & $M$ & 39 & Alentejo & MDR & NA & $\mathrm{R}$ & $\mathrm{R}$ & NA & NA & NA & NA & NA & NA & NA & NA & NA & NA & NA & \\
\hline S308144 & 2016 & & $M$ & 41 & LTV & XDR & $\mathrm{R}$ & $\mathrm{R}$ & $\mathrm{R}$ & $\mathrm{R}$ & $\mathrm{R}$ & $\mathrm{R}$ & $\mathrm{S}$ & $\mathrm{R}$ & $\mathrm{R}$ & $\mathrm{R}$ & $\mathrm{S}$ & $\mathrm{R}$ & $S$ & $\mathrm{~S}$ & 3 \\
\hline S309968 & 2016 & & $\mathrm{~F}$ & 41 & North & MDR & $\mathrm{R}$ & $\mathrm{R}$ & $\mathrm{R}$ & S & S & S & S & S & $\mathrm{S}$ & S & S & S & S & S & 4 \\
\hline S310368 & 2016 & & $M$ & 42 & North & XDR & $\mathrm{R}$ & $\mathrm{R}$ & $\mathrm{R}$ & $\mathrm{R}$ & $\mathrm{R}$ & $\mathrm{R}$ & S & $\mathrm{R}$ & $\mathrm{R}$ & $\mathrm{R}$ & S & $\mathrm{R}$ & S & S & 3 \\
\hline S312205 & 2016 & & $F$ & 63 & LTV & MDR & S & $\mathrm{R}$ & $\mathrm{R}$ & S & $\mathrm{R}$ & $\mathrm{R}$ & $\mathrm{R}$ & $\mathrm{R}$ & $\mathrm{S}$ & S & S & $\mathrm{R}$ & S & S & 1 \\
\hline S314371 & 2016 & & $M$ & 15 & LTV & MDR & $\mathrm{R}$ & $\mathrm{R}$ & $\mathrm{R}$ & $\mathrm{R}$ & $\mathrm{S}$ & S & S & $\mathrm{R}$ & $\mathrm{S}$ & S & S & S & S & S & 6 \\
\hline \multirow[t]{2}{*}{ S324134 } & 2016 & & M & 61 & Centre & MDR & $\mathrm{R}$ & $\mathrm{R}$ & $\mathrm{R}$ & $\mathrm{R}$ & $\mathrm{R}$ & S & S & S & $\mathrm{S}$ & S & S & $\mathrm{R}$ & S & S & \\
\hline & 2016 & $\begin{array}{l}\text { No culture } \\
\text { isolation }\end{array}$ & $\mathrm{F}$ & 45 & LTV & MDR & $\mathrm{R}$ & $\mathrm{R}$ & $\mathrm{R}$ & $\mathrm{R}$ & $\mathrm{R}$ & NA & NA & NA & NA & NA & NA & NA & NA & NA & \\
\hline S326551 & 2016 & & M & 40 & LTV & MDR & $\mathrm{R}$ & $\mathrm{R}$ & $\mathrm{R}$ & $\mathrm{R}$ & $\mathrm{R}$ & S & S & S & S & S & S & $\mathrm{S}$ & S & S & \\
\hline S327889 & 2016 & & $\mathrm{~F}$ & 22 & LTV & XDR & $\mathrm{R}$ & $\mathrm{R}$ & $\mathrm{R}$ & $\mathrm{R}$ & $\mathrm{R}$ & $\mathrm{R}$ & S & $\mathrm{R}$ & $\mathrm{R}$ & $\mathrm{R}$ & S & $\mathrm{R}$ & S & S & 3 \\
\hline S320857 & 2016 & & M & 43 & LTV & MDR & $\mathrm{R}$ & $\mathrm{R}$ & $\mathrm{R}$ & $\mathrm{R}$ & $\mathrm{R}$ & S & S & S & $\mathrm{S}$ & S & S & $\mathrm{s}$ & S & S & \\
\hline S316569 & 2016 & & M & 54 & North & MDR & $\mathrm{R}$ & $\mathrm{R}$ & $\mathrm{R}$ & S & $\mathrm{s}$ & $\mathrm{R}$ & $\mathrm{R}$ & S & $\mathrm{S}$ & S & S & $\mathrm{R}$ & S & S & \\
\hline S326782 & 2016 & & M & 62 & North & MDR & $\mathrm{R}$ & $\mathrm{R}$ & $\mathrm{R}$ & S & $\mathrm{S}$ & S & S & $\mathrm{R}$ & S & S & S & S & S & S & 3 \\
\hline S340248 & 2016 & & M & 20 & LTV & MDR & $\mathrm{R}$ & $\mathrm{R}$ & $\mathrm{R}$ & S & $\mathrm{R}$ & S & S & $\mathrm{R}$ & S & S & S & S & S & S & 2 \\
\hline TB31393 & 2016 & & $\mathrm{~F}$ & 40 & LTV & MDR & $\mathrm{R}$ & $\mathrm{R}$ & $\mathrm{R}$ & S & $\mathrm{S}$ & S & S & S & S & S & S & S & S & S & \\
\hline S332846 & 2016 & & $M$ & 70 & Centre & MDR & $\mathrm{R}$ & $\mathrm{R}$ & $\mathrm{R}$ & S & $\mathrm{R}$ & S & S & S & S & S & S & S & S & S & \\
\hline S347401 & 2016 & & $\mathrm{~F}$ & 20 & LTV & MDR & $\mathrm{R}$ & $\mathrm{R}$ & $\mathrm{R}$ & $\mathrm{R}$ & S & S & S & $\mathrm{R}$ & S & S & S & S & S & S & \\
\hline ACC & 2016 & & $\mathrm{~F}$ & 40 & LTV & MDR & $\mathrm{R}$ & $\mathrm{R}$ & $\mathrm{R}$ & S & $\mathrm{R}$ & S & S & $\mathrm{R}$ & S & S & S & S & S & S & 2 \\
\hline S333605 & 2016 & & M & 62 & North & MDR & $\mathrm{R}$ & $\mathrm{R}$ & $\mathrm{R}$ & S & S & S & S & $\mathrm{R}$ & S & S & S & S & S & $\mathrm{S}$ & 3 \\
\hline S348387 & 2016 & & M & UNK & Centre & MDR & S & $\mathrm{R}$ & $\mathrm{R}$ & S & $\mathrm{S}$ & S & S & S & S & S & S & S & S & S & \\
\hline S352139 & 2017 & & $\mathrm{~F}$ & 22 & Centre & MDR & $\mathrm{R}$ & $\mathrm{R}$ & $\mathrm{R}$ & $\mathrm{R}$ & $\mathrm{R}$ & S & S & S & S & S & S & S & S & S & 6 \\
\hline S375001 & 2017 & & $\mathrm{~F}$ & 22 & Centre & MDR & S & $\mathrm{R}$ & $\mathrm{R}$ & $\mathrm{R}$ & S & S & S & $\mathrm{R}$ & S & S & S & S & S & S & 5 \\
\hline S374686 & 2017 & & $\mathrm{~F}$ & 34 & LTV & MDR & S & $\mathrm{R}$ & $\mathrm{R}$ & $\mathrm{R}$ & $\mathrm{R}$ & S & S & $\mathrm{R}$ & S & S & S & S & S & S & \\
\hline S381277 & 2017 & & $M$ & 30 & North & MDR & $\mathrm{R}$ & $\mathrm{R}$ & $\mathrm{R}$ & $\mathrm{R}$ & S & S & S & S & S & S & S & S & S & S & \\
\hline TB33470 & 2017 & & M & 52 & LTV & MDR & S & $\mathrm{R}$ & $\mathrm{R}$ & $\mathrm{R}$ & $\mathrm{R}$ & S & S & $\mathrm{R}$ & S & S & S & S & S & S & 1 \\
\hline S389865 & 2017 & & $\mathrm{~F}$ & 59 & North & MDR & $\mathrm{R}$ & $\mathrm{R}$ & $\mathrm{R}$ & $\mathrm{R}$ & $\mathrm{R}$ & S & S & S & S & S & S & S & S & S & \\
\hline S387683 & 2017 & & M & 56 & LTV & XDR & $\mathrm{R}$ & $\mathrm{R}$ & $\mathrm{R}$ & $\mathrm{R}$ & $\mathrm{R}$ & $\mathrm{R}$ & S & $\mathrm{R}$ & $\mathrm{R}$ & $\mathrm{R}$ & S & $\mathrm{R}$ & S & S & 3 \\
\hline S399045 & 2017 & & $\mathrm{~F}$ & 41 & North & MDR & $\mathrm{R}$ & $\mathrm{R}$ & $\mathrm{R}$ & S & S & S & S & $\mathrm{R}$ & $\mathrm{R}$ & $\mathrm{R}$ & S & $\mathrm{S}$ & S & S & \\
\hline S396397 & 2017 & & $M$ & 51 & LTV & XDR & $\mathrm{R}$ & $\mathrm{R}$ & $\mathrm{R}$ & $\mathrm{R}$ & $\mathrm{R}$ & $\mathrm{R}$ & $\mathrm{R}$ & $\mathrm{R}$ & $\mathrm{S}$ & S & S & $\mathrm{R}$ & S & S & 1 \\
\hline S399986 & 2017 & & $M$ & 58 & LTV & MDR & $\mathrm{R}$ & $\mathrm{R}$ & $\mathrm{R}$ & $\mathrm{R}$ & S & S & S & $\mathrm{R}$ & $\mathrm{R}$ & $\mathrm{R}$ & S & $S$ & S & S & 3 \\
\hline \multirow[t]{2}{*}{ TB34192 } & 2017 & & $M$ & 62 & LTV & MDR & $\mathrm{R}$ & $\mathrm{R}$ & $\mathrm{R}$ & S & S & S & S & $\mathrm{R}$ & $S$ & S & S & $\mathrm{S}$ & S & S & 2 \\
\hline & 2017 & Only LPA & $M$ & 54 & North & MDR & NA & $\mathrm{R}$ & $\mathrm{R}$ & NA & NA & NA & NA & NA & NA & NA & NA & NA & NA & NA & \\
\hline
\end{tabular}

MDR: multidrug-resistant; XDR: extensively drug-resistant; TB: tuberculosis; STR: streptomycin; INH: isoniazid; RMP: rifampicin; EMB: ethambutol; PZA: pyrazinamide; AMI: amikacin; CAP: capreomycin; ETI: ethionamide; MOX: moxifloxacin; OFL: ofloxacin; LIN: linezolid; KAN: kanamycin; CIC: cycloserine; PAS: para-aminosalicylic acid; M: male; F: female; LTV: Lisbon and Tagus Valley; R: resistant; S: sensitive; NA: not available; LPA: line-probe assay; UNK: unknown. 
North region and the remaining clusters were mainly from LTV strains. Regarding the MDR-TB strains isolated in 2015, three clusters were found, with a clustering rate of $55.0 \%$, ranging from two to five strains. All the clustered strains were from the LTV region with the exception of one strain that belonged to a patient from the North region. In 2016, only two clusters were found with two and five strains, with a clustering rate of $38.9 \%$. The minor cluster was from a mother/child and the larger included strains from the LTV and the North regions. Finally, in 2017, two clusters were identified with two strains each, all from the LTV region, corresponding to a clustering rate of $36.4 \%$.

When linking the epidemiological and the molecular data, we did not find a good agreement. After adjustment for confirmed epidemiological links, the overall cluster rate (2014-2017) decreased from 63.4\% to $14.9 \%$.

This study has a limitation related to the possible heterogeneity of the epidemiological enquiries. However, it has the strength of collecting all MDR-TB samples in the country for 4 years to be analysed in the NRL.

We observed, in the studied period, a decreasing tendency both in the number of MDR-TB cases and the clustering rates, despite a poor agreement between laboratory and epidemiological data. The centralisation of the MDR-TB cases in reference centres seems to be effective, although there is a need for a better molecular tool, with higher discriminatory power, and better inclusion of epidemiological data when discussing these clusters.

Rita Macedo $\oplus^{1}$ and Raquel Duarte ${ }^{2}$

${ }^{1}$ National Reference Laboratory for Mycobacteria, Dept of Infectious Diseases, National Institute of Health, Lisbon, Portugal. ${ }^{2}$ General Health Directorate, Lisbon, Portugal.

Correspondence: Rita Macedo, National Reference Laboratory for Mycobacteria, Dept of Infectious Diseases, National Institute of Health, Avenida Padre Cruz, 1649-016 Lisbon, Portugal.

E-mail: rita.macedo@insa.min-saude.pt

Received: Sept 072018 | Accepted after revision: Jan 022019

Conflict of interest: None declared.

\section{References}

1 World Health Organization. Global Tuberculosis Report 2018. Geneva, WHO, 2018. Available from: www.who.int/ tb/publications/global_report/en/

2 Portuguese General Health Directorate. Tuberculose em Portugal - Desafios e Estratégias - 2018 [Tuberculosis in Portugal - Challenges and Strategies - 2018]. Lisbon, Portuguese General Health Directorate, 2018. Available from: www.dgs.pt/documentos-e-publicacoes/tuberculose-em-portugal-desafios-e-estrategias-2018-.aspx

3 Rito T, Matos C, Carvalho C, et al. A complex scenario of tuberculosis transmission is revealed through genetic and epidemiological surveys in Porto. BMC Infect Dis 2018; 18: 53.

4 Portuguese General Health Directorate. Testes de Sensibilidade aos Antituberculosos de 2a Linha [2nd Line Antitubercular Sensitivity Tests]. Lisbon, Portuguese General Health Directorate, 2007. Available from: http://nocs. pt/testes-de-sensibilidade-aos-antituberculosos-de-2a-linha/

5 Portugal I, Barreiro L, Vultos T, et al. Epidemiologia molecular de Mycobacterium tuberculosis em Lisboa [Molecular epidemiology of Mycobacterium tuberculosis in Lisbon]. Rev Port Pneumol 2008; 14: 239-259.

6 Macedo R, Antunes AF, Villar M, et al. Multidrug and extensively drug-resistant tuberculosis in Lisbon and Vale do Tejo, Portugal, from 2008 to 2010. Int J Mycobacteriol 2012; 1: 131-136. 